\title{
A Study on Image Processing of Log Inner Decay Derived from Stress Wave Testing Based on MATLAB
}

\author{
Wenshu Lin and Jinzhuo $\mathrm{Wu}^{*}$ \\ College of Engineering and Technology, Northeast Forestry University, \\ Harbin 150040, China \\ linwenshu@126.com, *Corresponding author:jinzhuo2009@gmail.com
}

\begin{abstract}
This paper deals about image processing of log internal decays using MATLAB technique. Specifically, image enhancement, image edge detection and decay area extraction and calculation on the two-dimension image of log inner decays that were derived by using the non-destructive inspection technique of stress wave were conducted. The results showed that the testing image of stress wave can visually reflect wood inner decay, and the image resolution can also be improved by using the functions of image enhancement and edge detection in MATLAB software. The detection precision of stress wave is closely associated with the ratio of log internal decay area to tested wood crosssectional area. When the ratio between wood actual inner decay area and tested log cross-sectional area increased from $4.77 \%$ to $45.52 \%$, the relative error between tested decay area and actual decay area reduced from $16.42 \%$ to $7.39 \%$. The study has provided an advanced method to judge the degree of wood inner decay for most forest practioners and researchers. Through the image processing and computing on the images derived by stress wave testing, the accuracy of logs internal decay discrimination can be significantly improved.
\end{abstract}

Keywords: stress wave, non-destructive detection, log, image processing, MATLAB

\section{Introduction}

Wood non-destructive detection technology is a newly arisen technology after 1950s, which can be used to test wood growth characteristics, physical properties, mechanical properties, and structural wood component defects. With the development of electron, optics, and computer technique, many wood non-destructive technologies have been successfully applied in wood properties and defects, such as x-ray testing, ultrasonic testing, nuclear magnetic resonance testing, microwave testing, and stress wave testing [1]. Compared to other non-destructive detection technologies (such as industry CT, Xray), the stress wave non-destructive detection equipment is easy to take and low cost. In addition, stress wave testing has no damage on wood itself and is not affected by the size and shape of wood samples. There is no need to apply couple mixture between sensor and tested wood samples. Therefore, stress wave testing has been widely applied in wood defect judgments and measurement of wood mechanical properties [2-5].

Wood inner defects include decay, knot, crack, holes, etc., among which inner decay is one of the most important wood defects, which can have negative impacts on wood quality. The shape and size of log inner decays can seriously affect the log grade in tree stem bucking. Therefore, how to apply an advanced method to determine the precision rate of log inner decay has becomes an important issue for forestry researchers in China. In this study, the section image of log inner decay tested by stress wave was processed by using the image processing toolbox in MATLAB (Matrix laboratory). Now, MATLAB software has more than 30 toolboxes and has multiple functions, such as neural network, optimization, wavelet 
analysis, signal processing, image processing, control system, differential equation, robust, fuzzy logic, and system identification [6]. The two-dimensional color image of wood decay was obtained by testing a log sample using stress wave instrument. The color image was saved in a computer through format conversion, and then MATLAB software was applied to extract and calculate the decay area and perform image processing and edge testing. The corresponding image pattern change before and after processing was used to determine log internal defects and thereby improve the reliability of defect detection inside the log.

\section{Stress Wave Testing}

\subsection{Testing System}

The stress wave testing instrument ARBOTOM imported from Germany was applied to test wood inner conditions according to the principle of measurement of stress wave propagation time. The log inner defect information can be obtained by ARBOTOM because of highly correlated relationships between stress waves propagate velocity within the $\log$ and wood density. In this study, a total of 6 sensors was fixed around the $\log$ and used to measure the related travel time of stress wave, and then ZDPC_PROG software was employed to analyze the processing data. Then, the propagate velocity of stress wave, propagate path, and section shape of log decay can be obtained in Figure 1. Before testing the wood sample using stress wave, it is necessary to input various parameters, including the number of sensors, sensor location, distance and the units of distance, sensor height, PC interface, the filtering method of measurement value, and species names, etc.

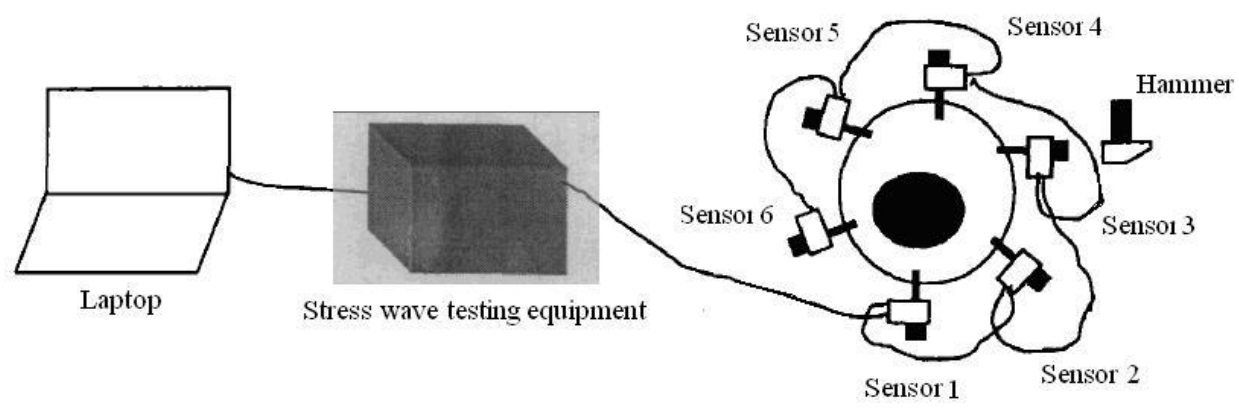

Figure 1. Stress Wave Testing System for Log Inner Decay

\subsection{Testing System}

The experimental materials are the dominant forest species in the Northeast area of China. Both conifers and broad-leaved trees were selected for the experiment. The conifers include korean pine, spruce, fir, larch, and broad-leaved trees include basswood, populus tomentosa, manchurian ash, juglans, quercus mongolica, and elm. Since log shape is approximate ellipse, cross-sectional area was computed in accordance with the elliptical formula (Table 1). The experiment was conducted in the indoor environment with room temperature at $20{ }^{\circ} \mathrm{C}$ and relative air humidity at $72 \%$.

Table 1. Description of the General Characteristics of the Ten Logs

\begin{tabular}{|c|c|c|c|c|c|}
\hline $\begin{array}{c}\text { Serial } \\
\text { number }\end{array}$ & Species & $\begin{array}{c}\text { Humidity } \\
\%\end{array}$ & $\begin{array}{c}\text { Longer } \\
\text { diameter, } \mathrm{cm}\end{array}$ & $\begin{array}{c}\text { Shorter } \\
\text { diameter, } \mathrm{cm}\end{array}$ & $\begin{array}{c}\text { Cross- } \\
\text { sectional } \\
\text { area, } \mathrm{cm}^{2}\end{array}$ \\
\hline
\end{tabular}




\begin{tabular}{cccccc}
\hline 1 & spruce & 10.2 & 20 & 19 & 296.73 \\
2 & fir & 9.7 & 23 & 23 & 415.26 \\
3 & larch & 12.5 & 22 & 21 & 362.67 \\
4 & korean pine & 13.6 & 21 & 20 & 329.7 \\
5 & basswood & 10.9 & 24 & 20 & 376.8 \\
6 & populus tomentosa & 11.5 & 25 & 22 & 431.75 \\
7 & manchurian ash & 16.3 & 24 & 21 & 395.64 \\
8 & juglans & 11.2 & 21 & 21 & 346.18 \\
9 & quercus mongolica & 15.8 & 26 & 25 & 510.25 \\
10 & elm & 9.8 & 32 & 29 & 728.48 \\
Average & - & 12.15 & 23.80 & 22.10 & 419.35 \\
\hline
\end{tabular}

\subsection{Testing Image}

During stress wave testing, the sensors were firstly hanged on the log in sequence and the distance between each pair of sensors was input in the software. The sensors were then hit by a hammer in sequence, and the log section image will be displayed in the attached computer. It was apparent from the tested section image that when the log inner decay exists, the image color of the section will change to some extent (green represents the log without decay and red represents the log with decay). Therefore, we can judge whether there is log decay based on the color of the image. The specific testing results by two species are shown in Figure 2 and Figure 3, respectively

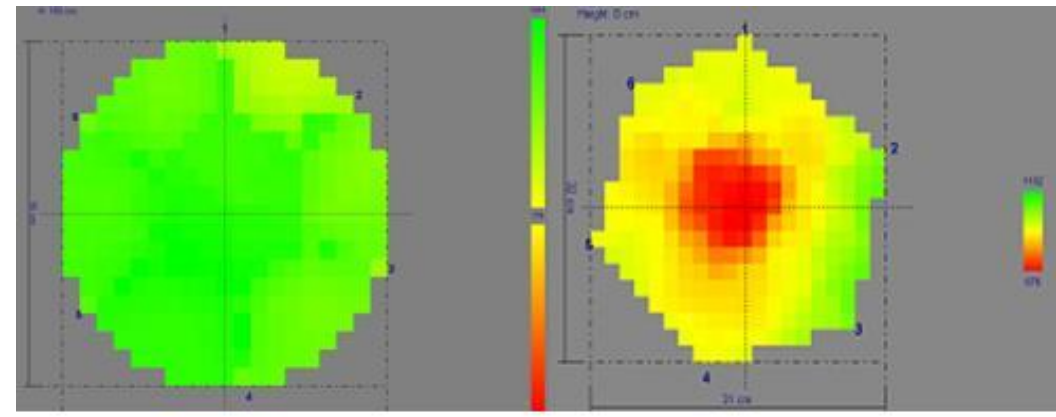

(a)

(b)

Figure 2. Korean Pine Log Cross-section Derived by Stress Wave Testing: (a) Log without Decay (b) Log with Decay.

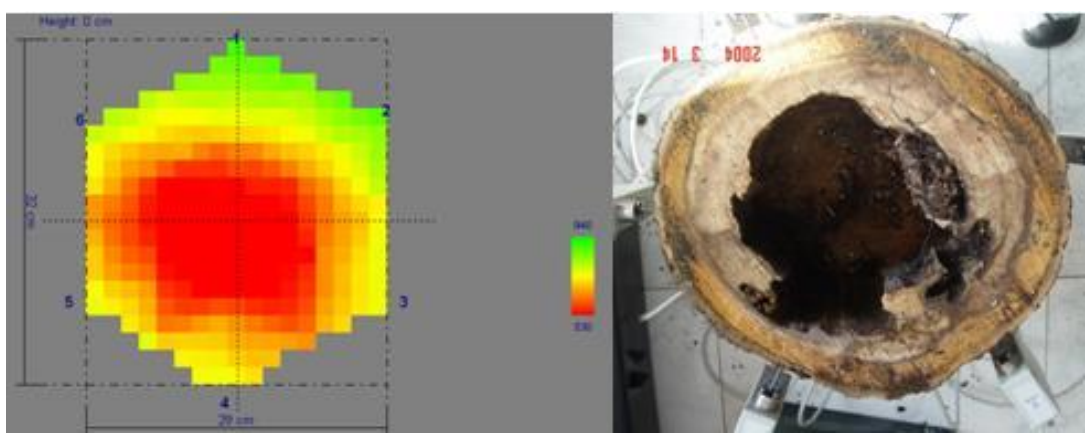

Figure 3. Elm Wood-section Image by Stress Wave Detection and the Actual Log Cross-section.

Figure 2 and Figure 3 showed that stress wave can be used to test the existence of $\log$ decay. The scale of inner decay area has significant impact on the precision of 
the image of stress wave detection. In a certain range, the larger the ratio between actual decay areas and tested log cross section areas, the more obvious the image. However, the detection effects (e.g., outline of log inner decay and area identification) were not well enough using the section image produced by the attached software in stress wave detection instrument. The reason may be that the stress waves propagating through the cross-section of a log will be subjected to a number of factors, such as the shape and scale of decay, the quantity of sensors, calculation algorithm of software, wood species, and wood moisture content [7]. Therefore, effective and further research on image processing should be taken to reduce the testing error and improve the quality and accuracy of testing image. This study deals image enhancement and edge detection processing, log inner decay calculation and measurement by using the MATLAB software.

\section{Log Image Processing}

\subsection{Image Enhancement Processing}

The purpose of image enhancement is to improve the quality of the image. For a given image, depending on the image fuzzy condition and application situation, image enhancement can be realized using special technology to highlight or weaken some irrelevance information in the image. So it can purposefully highlight the whole or part image characteristics which are helpful to improve the recognition capability, and also contribute to observers to analyze the information directly and clearly. Image enhancement of wood defect detection with MATLAB software will improve the defect resolution of the detection image. In the study, the original color image was firstly converted to gray image and then histogram equalization was applied to the gray image (Figure 4). The images before and after gray-scale transformation, and before and after true color image enhancement processing were illustrated in Figure 5, and Figure 6, respectively.

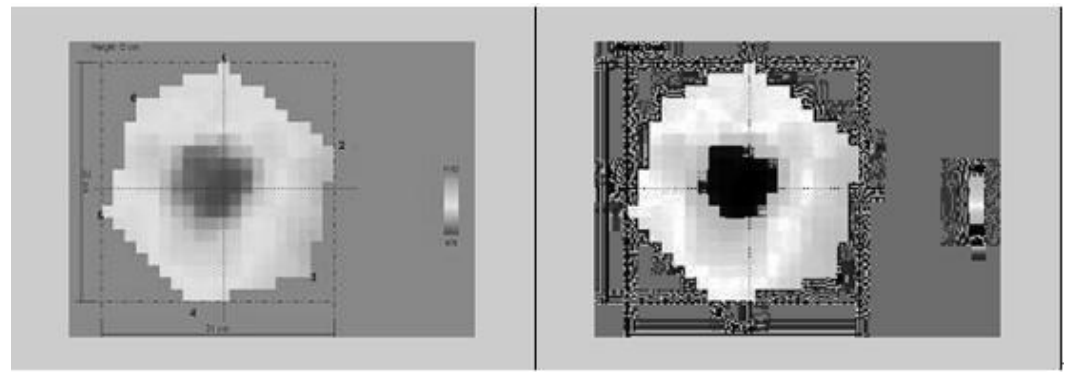

Figure 4. The Results Before and After Histogram Equalization

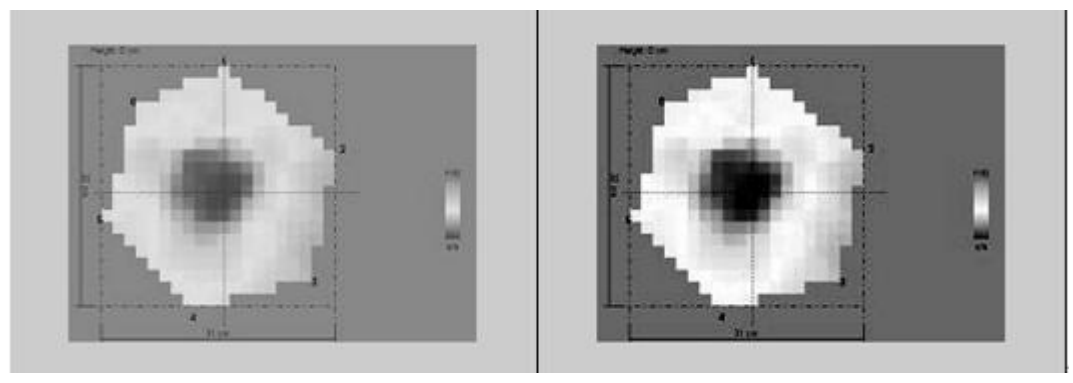

Figure 5. The Image Before and After Gray-scale Transformation 


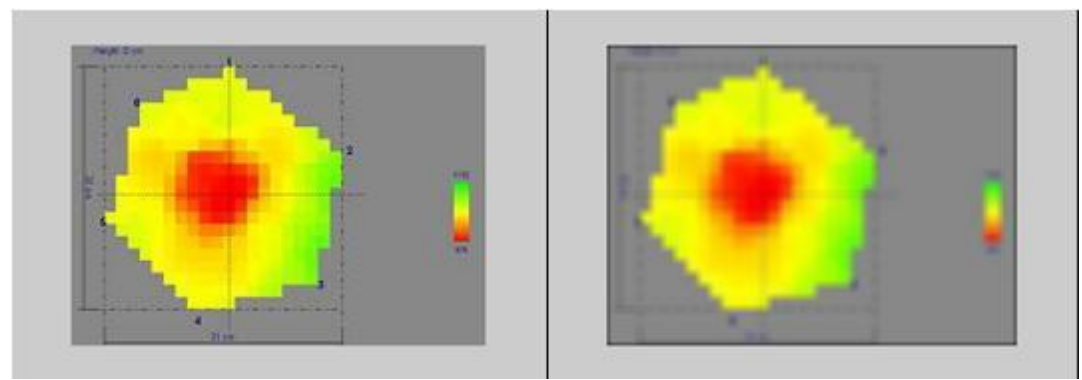

\section{Figure 6. The Image Before and After True Color Image Enhancement Processing}

The image processing Toolbox in MATLAB provides a stable, well-supported software environment for addressing a broad range of applications in digital image processing. Due to the fast computation speed and the provided scientific algorithms, the image processing functions in MATLAB can meet the requirements of wood image defect detection and help improve the accuracy of wood defect detection. Although there are many image enhancement methods, different image enhancement method should be applied in consideration of the specific purpose of image enhancement. The enhanced tested image showed that the image become visible and distinct compared to the gray-level image and equalized image. The shape of the histogram equalization is better than the original shape of the histogram. Wood inter decay can be easily identified through color enhancement.

\subsection{Edge Detection and Processing}

The basic characteristic of an image is the edge. An edge can be defined as a set of contiguous pixel positions where an abrupt change of intensity (gray or color) values occur. Edge mostly exists between target and target, target and background, region and region, primitive and primitive. Therefore, it is the most important character of image segmentation, important information sources of textural feature and foundation of shape feature [8]. Edge detection is a fundamental tool used in most image processing applications to obtain information from the grames as a precursor step to feature extraction and object segmentation. There are many ways to perform edge detection. However, the most may be grouped into two categories, gradient and Laplacian. The gradient method detects the edges by looking for the maximum and minimum in the first derivative of the image. The Laplacian method searches for zero crossings in the second derivative of the image to find edges. Examples of gradient-based edge detectors are Roberts, Prewitt, and Sobel operators.

In this study, the edge refers to the contour of the inner defect within a section image, such as decay and hole. The shape of the defect was determined by using edge detection operator. MATLAB image toolbox provides an edge function which can be used in edge detection of gray image. The function BW=edge (I, 'method') was used to process edge detection of the image, where I represents the saved image. A binary image that is as big as I will then be returned in which 1 represents dot of edge and 0 represents dot of non-edge. The Prewitt, Sobel and Log method were used to process edge detection of the log section image tested by stress wave. The methods are very important to qualitatively analyze the wood inner defect. Since the image produced by stress wave detection was a two-dimension real-color image, it should be converted to gray-level image, and then the three methods previously mentioned were applied to perform edge detection. The processed result showed that the location and shape of the wood inner defect was very clear, which met the requirements of image edge detection and improved the reliability of wood inner defects detection. The output results are shown in Figure 7. 


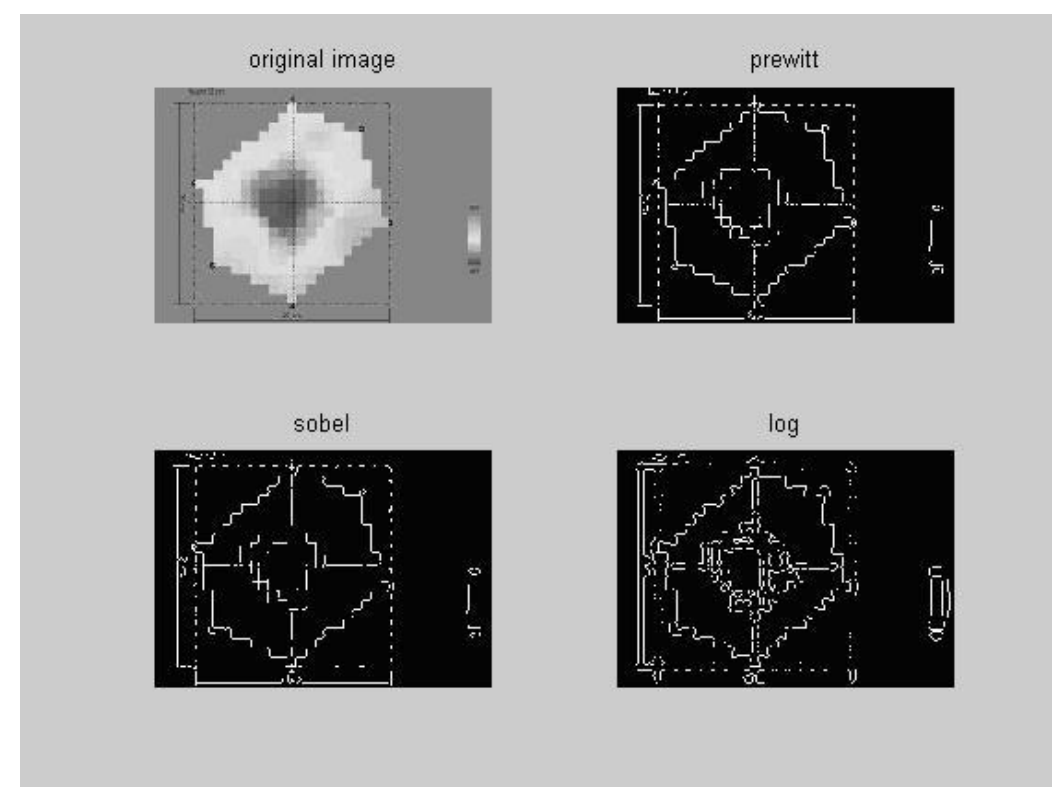

Figure 7. Edge Detection Results by using Different Algorithms on the Log Cross-section

\section{Recognition and Calculation of Log Inner Decay}

After the wood defect image being processed, some steady features that are able to reflect the nature of the defect were selected as the basis for defect classification, identification and interpretation. Given the status of wood defect image processing, it is impossible to establish an expert knowledge base with all kinds of defect types. So the selection of wood defect characteristics mainly depends on the experimental results and analysis of the defects.

In this study, the extraction and calculation of log inner decay area were done by MATLAB software. Firstly, the defect image binarization was completed. Here, the defects were extracted. Secondly, morphological opening operation was applied to split the cross sectional area and the defect area (Figure 8). Based on the number of pixels of the splitted white area, the ratio of the number of defective pixels to the total number of pixels of the entire cross-section can be obtained. Since the shape of log cross-section can be approximately considered as ellipse, the ellipse area can be calculated based on the long axis and short axis of the ellipse. Finally, the real log inner decay area can be determined according to the pixel ratio and cross-section area of the log. 


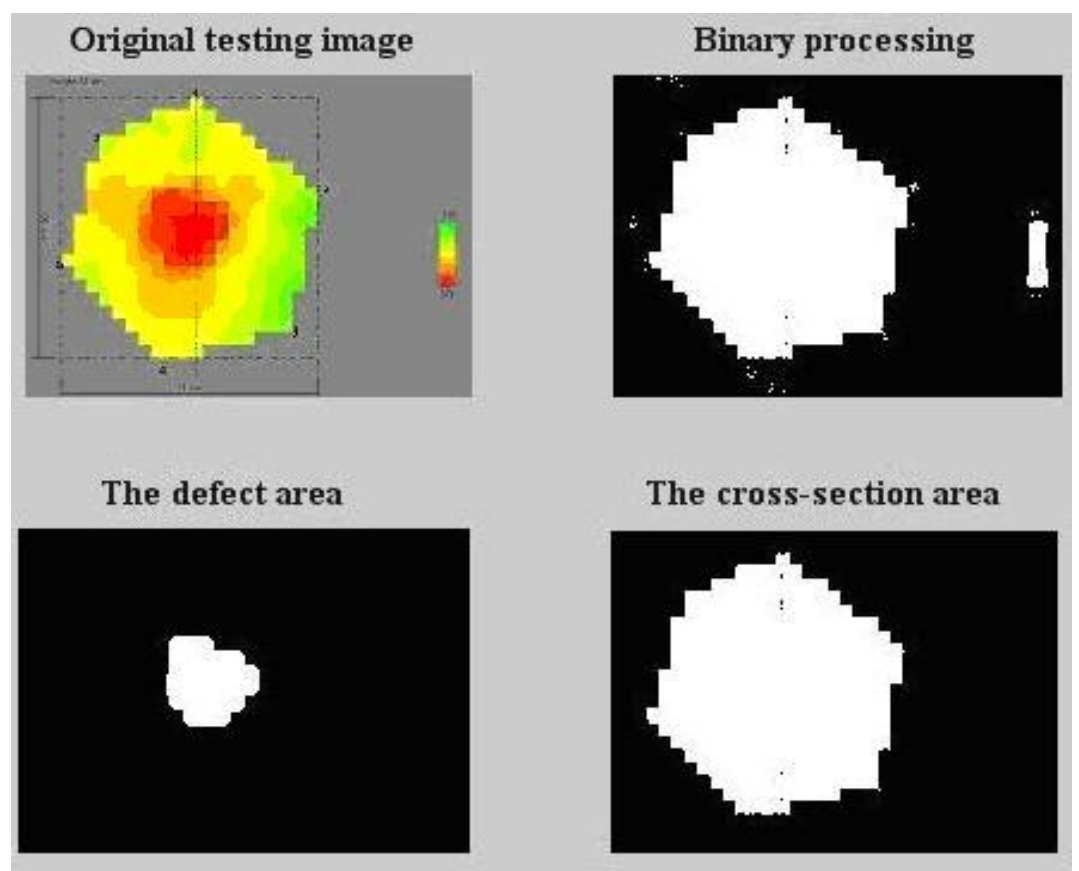

\section{Figure 8. Morphology Operation Processing Defects and Cross-sectional Area}

The results showed that after morphological processing, the number of defect pixels accounted for $9.78 \%$ of the total number of pixels of the cross section (the number of pixels in the defect area was 1,120 and the number of pixels of the cross section was 11,455). The log cross section area was $329.7 \mathrm{~cm}^{2}$ based on the real logs long and short axis $(21 \mathrm{~cm}$ and $20 \mathrm{~cm})$. Therefore, the inner decay area of the log was $32.23 \mathrm{~cm}^{2}$. To verify the scale error of the estimated log inner decay area, we made a comparison between the measured log inner decay area and the estimated log decay area produced by the testing image.

The actual log decay area was estimated as follows: The outline image of the log inner decay area was determined by using the magic tool in Photoshop in consideration of the arbitrary shape of decay area. After being processed, the image was saved as a new image file. The ERDAS IMAGEING software was used to export the new reserved image and the actual $\log$ decay area was statistically computed using the vector module. ERDAS IMAGEING is a remote sensing application with raster graphics editor abilities designed by ERDAS for geospatial applications. It has measurement, statistics, and editing functions. Taking Figure 3 as an example, the actual decay area obtained by using the software was $35.93 \mathrm{~cm}^{2}$. Therefore, the difference of $\log$ inner decay area between the detected and the actual was $3.7 \mathrm{~cm}^{2}$, and the correspondingly relative error was $10.29 \%$.

According to the above mentioned tested images and the method for calculating actual $\log$ decayed area, a total of $10 \operatorname{logs}$ were collected with detection images. The calculated and actual log inner decay area and the magnitude of the error value were summarized in Table 2. It is noted that when the ratio between the measured actual decayed area and log cross-sectional area increased from $4.77 \%$ to $45.52 \%$, the relative error between the the tested area and actual decayed area decreased from $16.42 \%$ to $7.39 \%$. Therefore, the relative error between the stress wave detection and the actual decay area decreased with the increase of log inner decay area. In addition, the shape of logs internal decay has somewhat negative impact on the detection error rate. The more regular of the shape of decay, the smaller the error rate. For example, the third log had irregular shape and the error rate was $18.32 \%$, however the tenth $\log$ has regular shape and the error rate was only $7.39 \%$. 
Table 2 The Decay Area of the Ten Logs

\begin{tabular}{cccccc}
\hline Log & $\begin{array}{c}\text { Real log decay } \\
\text { area/log cross- } \\
\text { section area, \% }\end{array}$ & $\begin{array}{c}\text { Decay area } \\
\text { of testing } \\
\text { image, } \mathrm{cm}^{2}\end{array}$ & $\begin{array}{c}\text { Decay area } \\
\text { of real log, } \\
\mathrm{cm}^{2}\end{array}$ & $\begin{array}{c}\text { Area } \\
\text { difference, } \\
\mathrm{cm}^{2}\end{array}$ & $\begin{array}{c}\text { Relativ } \\
\text { e area, } \\
\%\end{array}$ \\
\hline 1 & 4.77 & 11.83 & 14.15 & 2.19 & 16.42 \\
2 & 5.65 & 20.33 & 23.46 & 3.00 & 13.35 \\
3 & 6.92 & 20.5 & 25.10 & 4.47 & 18.32 \\
4 & 10.89 & 32.23 & 35.93 & 3.7 & 10.29 \\
5 & 11.02 & 36.85 & 41.52 & 4.54 & 11.25 \\
6 & 11.82 & 45.69 & 51.03 & 5.21 & 10.47 \\
7 & 12.42 & 44.91 & 49.14 & 3.56 & 8.61 \\
8 & 18.56 & 58.08 & 64.25 & 6.04 & 9.60 \\
9 & 23.96 & 111.3 & 122.26 & 10.83 & 8.96 \\
10 & 45.52 & 307.05 & 331.58 & 24.53 & 7.39 \\
Average & 0.15 & 68.88 & 75.84 & 6.97 & 11.47 \\
\hline
\end{tabular}

\section{Conclusions}

In this study, stress wave non-destructive testing technology was used to detect the internal decay of logs which are the main species in the northeastern region of China. Proper image processing was conducted on the tested images of the ten logs and the following conclusions can be drawn from the study:

(1) Stress wave detection system is able to reflect log inner decay visually, but the outline and area of the tested image can be easily affected by many other factors, therefore further image processing is necessary in order to accurately reflect the defect information.

(2) Through the image processing (i.e., image enhancement, image edge detection) using MATLAB software on the tested image derived by stress wave detection, the log inner decay can be easily identified and its shape and outline can be clearly recognized.

(3) Based on the analysis results, we found that there was a certain relative error between the testing image area and actual decayed area. The larger the ratio of the measured actual decayed area to the log cross-sectional area of the tested image, the smaller the relative error. In addition, the shape of logs internal decay has a certain impact on the detection error rate.

It is suggested that image processing should be combined with the analysis on the changes of stress wave propagation parameters (travel time, velocity, and elastic modulus, etc.,) while applying stress waves to detect and justify log inner decay. The usage of graphics aided software such as MATLAB will also help improve the reliability of defect detection. In addition, in order to improve the detection accuracy of stress wave testing, only processing the detection image is not enough, because the usage of stress wave detecting internal log defects can be affected by various factors, such as the number of sensors, different kinds of wood materials, wood internal moisture content, density, and other factors. They will affect the detection precision of stress waves testing results. Therefore, further research on improving the accuracy of stress wave detecting of logs internal defects was still necessary.

\section{Acknowledgements}

The authors would like to acknowledge the support of the Fundamental Research Funds for the Central Universities (DL13BB19) and the Excellent Research Projects for Returned Overseas Scholars (415003). 


\section{References}

[1]. L. H. Wang, X. C. Yang, and K. H. Xu, "Current situation of research on the non-destructive testing technique for wood defects", Forest Engineering, vol. 27, no. 3, (2002), pp. 35-38.

[2]. X. C. Yang and L. H. Wang, "Gain and analysis of two-dimensional images of interior decay of logs with stress wave method", Forest Science, vol. 43, no. 11, (2007), pp. 93-97.

[3]. R. J. Ross and J. C. Ward, "A TenWolde, Stress wave nondestructive evaluation of wet wood", Forest Products Journal, vol. 44, vol. 7/8, (1994), pp. 79-83.

[4]. B. K. Brashaw, X. P. Wang, R. J. Ross and R. F. Pellerin, "Relationship between stress wave velocities of green and dry veneer", Forest Products Journal, vol. 54, no. 6, (2004), pp. 85-89.

[5]. F. G. Wagner, G. M. Thomas, L. H. Daryle, R. C. Raini and B. Todd, "Stress-wave analysis of DouglasFir logs for veneer properties”, Forest Products Journal, vol. 50, no. 4, (2000), pp. 49-52.

[6]. W. M. Tu, Y. G. Wei and S. M. Shi, "Application of MATLAB to digital image processing”, Control and Managementl, vol. 23, no. 6, (2007), pp. 299-301.

[7]. Y. P. Chen, X. Y. Liu, H. Li, Y. Han, D. Q. Li and T. Zhang, "Research on stress wave tomography of spruce logs with artificial defects under different number sensors", Forest Science, vol. 48, no. 3, (2012), pp. 97-101.

[8]. Y. Q. Zhang and F. R. Meng, "Application of MATLAB in image edge detection”, Application Research of Computers, vol. 6, (2004), pp. 144-146.

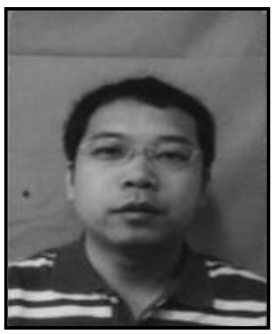

\section{Authors}

Wenshu Lin earned a B.E. degree and a M.S. degree in Forest Engineering from Northeast Forestry University, Harbin, P. R. China in 2003 and 2005, respectively. In 2011, he received a Ph.D. degree in Forest Resources Science from West Virginia University, USA. He is a Member of Forest Products Society and Society of Wood Science and Technology. Dr. Lin had several publications in esteemed journals such as Forest Products Journal, Wood and Fiber Science, and Computers and Electronics in Agriculture. Currently, he is an associate professor at Northeast Forestry University, Harbin, P. R. China. His major research interests include nondestructive testing and evaluation of wood, wood utilization and production improvement, and computer simulation and system modeling.

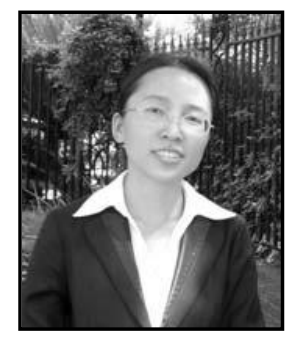

Jinzhuo Wu earned her B.E. degree and M.S. degree in Forest Engineering from Northeast Forestry University, Harbin, P. R. China in 2003 and 2005, respectively. In 2010, she obtained her $\mathrm{Ph} . \mathrm{D}$. degree in Forest Resources Science from West Virginia University, USA. She is a Member of Forest Products Society. Dr. $\mathrm{Wu}$ had several publications in esteemed journals such as Forest Products Journal, Wood and Fiber Science, Canadian Journal of Forest Research, and Journal of Agriculture and Resources Economics. 
International Journal of Signal Processing, Image Processing and Pattern Recognition Vol. 8, No. 2 (2015) 